University of Nebraska - Lincoln

DigitalCommons@University of Nebraska - Lincoln

USDA National Wildlife Research Center - Staff Publications
U.S. Department of Agriculture: Animal and Plant Health Inspection Service

2009

\title{
Evaluation of an Epitope-Blocking Enzyme-Linked Immunosorbent Assay for the Detection of Antibodies to Influenza A Virus in Domestic and Wild Avian and Mammalian Species
}

\author{
Heather J. Sullivan \\ USDA/APHIS/WS National Wildlife Research Center, Heather.Sullivan@aphis.usda.gov \\ Bradley J. Blitvich \\ lowa State University, Department of Veterinary Microbiology and Preventive Medicine \\ Kaci K. VanDalen \\ USDA/APHIS/WS National Wildlife Research Center, kaci.vandalen@aphis.usda.gov \\ Kevin T. Bentler \\ USDA-APHIS-Wildlife Services \\ Alan B. Franklin \\ United States Department of Agriculture, Wildlife Services, National Wildlife Research Center, \\ alan.b.franklin@aphis.usda.gov

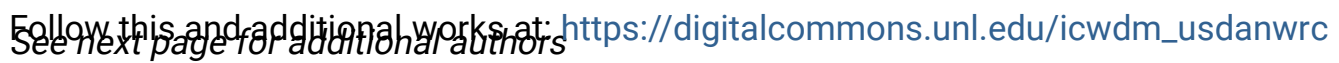 \\ Part of the Environmental Sciences Commons
}

Sullivan, Heather J.; Blitvich, Bradley J.; VanDalen, Kaci K.; Bentler, Kevin T.; Franklin, Alan B.; and Root, J. Jeffrey, "Evaluation of an Epitope-Blocking Enzyme-Linked Immunosorbent Assay for the Detection of Antibodies to Influenza A Virus in Domestic and Wild Avian and Mammalian Species" (2009). USDA National Wildlife Research Center - Staff Publications. 975.

https://digitalcommons.unl.edu/icwdm_usdanwrc/975

This Article is brought to you for free and open access by the U.S. Department of Agriculture: Animal and Plant Health Inspection Service at DigitalCommons@University of Nebraska - Lincoln. It has been accepted for inclusion in USDA National Wildlife Research Center - Staff Publications by an authorized administrator of DigitalCommons@University of Nebraska - Lincoln. 
Authors

Heather J. Sullivan, Bradley J. Blitvich, Kaci K. VanDalen, Kevin T. Bentler, Alan B. Franklin, and J. Jeffrey Root 


\title{
Evaluation of an epitope-blocking enzyme-linked immunosorbent assay for the detection of antibodies to influenza A virus in domestic and wild avian and mammalian species
}

\author{
Heather J. Sullivan ${ }^{\mathrm{a}, *, 1}$, Bradley J. Blitvich ${ }^{\mathrm{b}, 1}$, Kaci VanDalen ${ }^{\mathrm{a}}$, Kevin T. Bentler ${ }^{\mathrm{a}}$, \\ Alan B. Franklin ${ }^{\mathrm{a}}$, J. Jeffrey Root ${ }^{\mathrm{a}}$ \\ a United States Department of Agriculture, Wildlife Services, National Wildlife Research Center, 4101 LaPorte Ave., Fort Collins, CO 80521, USA \\ ${ }^{\mathrm{b}}$ Department of Veterinary Microbiology and Preventive Medicine, Iowa State University, Ames, IA 50011, USA
}

\section{Article history:}

Received 3 March 2009

Received in revised form 29 May 2009

Accepted 2 June 2009

Available online 11 June 2009

\section{Keywords:}

Avian influenza

bELISA

Influenza A virus

Serology

Wildlife

\begin{abstract}
A B S T R A C T
An epitope-blocking enzyme-linked immunosorbent assay (bELISA) was developed for the detection of antibodies to influenza A virus in taxonomically diverse domestic and wild vertebrate species. In contrast to the bELISAs published previously that require reagent production, manipulation by the end-user, or have not been evaluated for use with both mammalian and avian species, this assay is performed using commercially available recombinant nucleoprotein antigen and corresponding nucleoprotein-specific monoclonal antibody and has been shown to work with multiple avian and mammalian species. The efficacy of the bELISA as a serum screening assay was compared to the agar gel immunodiffusion (AGID) assay using 251 serum samples obtained from experimentally infected mallards (Anas platyrhynchos) and raccoons (Procyon lotor). The concordance between the AGID assay and bELISA was 94.1\% (95\% CI=89.9, 98.3) for raccoons, and 71.2\% (95\% $\mathrm{CI}=63.5,78.9)$ for mallards and $82.8 \%(95 \% \mathrm{CI}=78.2,87.3)$ overall. The bELISA was more sensitive than the AGID assay as demonstrated by the detection of antibodies to influenza A virus at earlier time points in experimental infection studies and at higher serial dilutions. The efficacy of the bELISA to monitor natural influenza A virus exposure was also compared to the AGID assay using an additional 745 serum samples from six avian species and six mammalian species. This bELISA provides a rapid, reliable, and inexpensive technique for large-scale surveillance of influenza $A$ virus exposure in taxonomically diverse vertebrate species.
\end{abstract}

Published by Elsevier B.V.

\section{Introduction}

Influenza viruses (family Orthomyxoviridae) are segmented, negative-sense, single-stranded RNA viruses that are classified into three types (A, B and C) based on the antigenic properties of their nucleoproteins (NP) and matrix proteins (Webster et al., 1992). Influenza $A$ viruses are further classified into different subtypes based on the antigenicity of their hemagglutinin (HA) and neuraminidase (NA) proteins (Spackman, 2008). To date, sixteen HA subtypes (H1-H16) and nine NA subtypes (N1-N9) of influenza A virus have been identified (Fouchier et al., 2005). While all known subtypes of influenza A virus are maintained in waterfowl (Fenner et al., 1993), these viruses have a broad host range that includes numerous avian and mammalian species (Palese and Shaw, 2007). Recently, the Asian strain of highly pathogenic avian influenza

\footnotetext{
* Corresponding author. Tel.: +1 970266 6123; fax: +1 9702666138 .

E-mail address: Heather.Sullivan@aphis.usda.gov (H.J. Sullivan).

${ }^{1}$ These authors contributed equally to this work.
}

(HPAI) H5N1 has attracted world-wide attention because it has been responsible for significant mortality in many domestic and wildlife species and has caused over 380 human cases of influenza A (H5N1) in Asia, Europe and Africa (WHO, 2008). Due to the rapid spread of HPAI H5N1, surveillance studies have been initiated to monitor the activity and spread of multiple influenza A viruses (Hall et al., 2008a,b; Paltrinieri et al., 2008; U.S. Interagency Strategic Plan, 2006).

One way to monitor influenza A virus activity in an area is to test serum samples from domestic and/or wild animals for the presence of antibodies. Traditionally, the serological tests suggested for large numbers of serum samples from domestic and/or wild animal studies have included the agar gel immunodiffusion (AGID) assay which detects the presence of antibodies to all influenza A viruses and the hemagglutination-inhibition (HI) test which detects antibodies to specific HA subtypes of influenza A viruses (OIE, 2008). The HI test is not typically utilized for large-scale influenza A virus serological surveys because many researchers do not have the facilities, reagents, or funds to conduct numerous $\mathrm{HI}$ assays for all $16 \mathrm{HA}$ subtypes. Due to its ability to detect antibodies to all influenza A 
viruses the AGID assay can be useful when screening large numbers of animals for the evidence of any influenza A virus exposure. However, while the AGID assay has been shown to work well with some species, such as poultry, it has not been thoroughly validated for use with others (Beard, 1970; OIE, 2008). Some species, such as mallards and swine, may not produce precipitating antibodies consistently, which are necessary for the AGID assay to perform properly (Beard, 1970; Higgins, 1989; Toth and Norcross, 1981). Due to possible constraints of the AGID assay various enzyme-linked immunosorbent assays (ELISAs) have been developed to detect antibodies to influenza A viruses from multiple species (Shafer et al., 1998; Starick et al., 2006; Wu et al., 2007). However, these ELISAs require extensive reagent production and manipulation, which may not be feasible for many researchers. The objective of this study was to develop an epitope-blocking ELISA (bELISA), using commercially available reagents that would provide research and diagnostic laboratories a rapid and inexpensive method to screen large numbers of serum samples from both domestic and wild animals for antibodies to all influenza A viruses. The utility of the bELISA was examined relative to the AGID assay by testing serum from known infected and control animals.

\section{Materials and methods}

\subsection{Reagent evaluation}

Direct ELISAs were performed to ensure that the commercial monoclonal antibody and nucleoprotein antigen could sufficiently bind and produce an enzymatic reaction measurable by a spectrophotometer. For the bELISA, these reagents were then independently optimized using well-characterized serum samples from experimentally infected and mock infected mallards (Anas platyrhynchos). All serum samples were tested in duplicate at a dilution of $1: 10$. The antigen was optimized using a range of concentrations from $1.6 \mu \mathrm{g} / \mathrm{ml}$ to $100 \mathrm{mg} / \mathrm{ml}$. The monoclonal antibody was optimized using a range of concentrations from $1.6 \mu \mathrm{g} / \mathrm{ml}$ to $50 \mathrm{mg} / \mathrm{ml}$. Concentrations that resulted in the greatest percent inhibition of monoclonal antibody binding were then used throughout the analyses.

\subsection{Serum samples}

A total of 251 serum samples from experimentally infected vertebrates and 745 serum samples collected in serological surveys belonging to 12 species (eight orders, 12 families) were used to evaluate the bELISA. Serum samples were obtained from raccoons (Procyon lotor), and mallards which were challenged experimentally with four different subtypes of influenza A virus. Eight raccoons were inoculated intranasally with $10^{5} \mathrm{EID}_{50}$ of influenza A virus subtype $\mathrm{H} 4 \mathrm{~N} 8$ (A/CK/AL/75[H4N8]), and four raccoons were inoculated intranasally with $10^{5}$ EID $_{50}$ of influenza A virus subtype H3N2 (A/Aichi/2/68 [H3N2]) (10). Twenty-three mallards were orally inoculated with $10^{6}$ EID $_{50}$ of influenza A virus subtype H4N6 (A/wildbird/PA/185996-06/07[H4N6]) and 27 mallards were orally inoculated with $10^{4}$ EID $_{50}$ of influenza A virus subtype H8N4 (A/ wildbird/CA/186243-18/06[H8N4]).

Additionally, sera were obtained from 215 birds and 38 mammals from Maryland in 2005 and 2006 and used to determine the diagnostic criterion for the bELISA. Avian species sampled from Maryland were as follows: Canada geese (Branta canadensis; $n=98$ ), great blue herons (Ardea herodias; $n=8$ ), a dark-eyed junco (Junco hyemalis; $n=1)$, mallards $(n=22)$, American white pelicans (Pelicanus erythrorhynchos; $n=20$ ), rock doves (Columba livia; $n=18$ ) and wood ducks (Aix sponsa; $n=48$ ) and mammalian species included American black bears (Ursus americanus; $n=10$ ), woodchucks (Mar- mota monax; $n=9$ ), raccoons ( $n=10)$, red fox (Vulpes vulpes; $n=1$ ), and white-tailed deer (Odocoileus virginianus; $n=8$ ). Sera were also obtained from 323 Canada geese sampled in Pennsylvania in 2003, 122 feral swine (Sus scrofa) sampled in Texas in 2005-2006 (Hall et al., 2008a), 16 raccoons sampled in a suburban area in Colorado in 2006 (Root et al., 2008), and 31 rock doves (C. livia) sampled in Idaho in 2008 and used to evaluate the efficacy of the bELISA in naturally exposed wildlife.

\subsection{Epitope-blocking enzyme-linked immunosorbent assay}

The bELISA platform used was similar to a published bELISA used for West Nile virus surveillance (Blitvich et al., 2003a,b) Briefly, the inner 60 wells of 96-well microtiter plates (Corning Inc., Corning, NY) were coated with $100 \mu$ l of recombinant influenza A virus nucleoprotein (Cat no. IMR-274; Imgenex, San Diego, CA) diluted optimally at approximately $143 \mathrm{mg} / \mathrm{ml}$ (prior to multiple freeze-thaw cycles) in carbonate-bicarbonate buffer $(50 \mathrm{mM}$ sodium carbonate, $50 \mathrm{mM}$ sodium bicarbonate, $\mathrm{pH}$ 9.6). The outer wells were filled with carbonate-bicarbonate buffer only. Coated plates were incubated overnight at $4{ }^{\circ} \mathrm{C}$ and washed four times with wash buffer (phosphate-buffered saline [PBS, pH 7.5] containing $0.1 \%$ Tween 20). Next, $200 \mu$ l of blocking buffer (PBS containing $5 \%$ skim milk) was added to each well and incubated for $40 \mathrm{~min}$ at $37^{\circ} \mathrm{C}$. After an additional four washes, $50 \mu \mathrm{l}$ of serum diluted 1:10 in blocking buffer was added in duplicate and incubated for $2 \mathrm{~h}$ at $37^{\circ} \mathrm{C}$, after which the wells were washed again four times. Next, $50 \mu \mathrm{l}$ of the influenza A virus nucleoprotein-specific monoclonal antibody clone A1 (Cat. No. MAB8257; Millipore Corp., Billerica, MA) optimally concentrated at $50 \mathrm{mg} / \mathrm{ml}$ in blocking buffer was added to each well and incubated for $1 \mathrm{~h}$ at $37^{\circ} \mathrm{C}$. Plates were again washed four times and $50 \mu \mathrm{l}$ of horseradish peroxidase-conjugated rabbit anti-mouse IgG (Invitrogen, Carlsbad, CA) was added at a concentration of $500 \mathrm{mg} / \mathrm{ml}$ to each well and incubated for $1 \mathrm{~h}$ at $37^{\circ} \mathrm{C}$, followed by four washes. Equal volumes of ABTS $\left(2,2^{\prime}\right.$ azino-bis[3-ethylbenzthiazoline-6-sulfonic acid]) and peroxidase solutions from the ABTS Microwell peroxidase substrate system (KPL, Gaithersburg, MD) were mixed, and $75 \mu$ l was added to each well. The optical density at a wavelength of $415 \mathrm{~nm}$ was determined with a spectrophotometer once negative controls reached an optical density of 0.30 . The percent inhibition of monoclonal antibody binding was calculated as $100-[(\mathrm{TS}-B) /(\mathrm{CS}-B)] \times 100$, where TS is the mean optical density of the test serum, CS is the mean optical density of the control serum (negative control serum from uninfected raccoons or mallards, depending on the species being tested), and $B$ is the background optical density. For estimation of the background optical density, four wells of each plate were incubated with coating buffer that did not contain influenza A virus nucleoprotein antigen. Serum samples were tested in duplicate, and at least four wells of negative control serum were included on each 96-well plate. The reagent and sample volumes and optical density of 0.3 (the negative control serum cut off) used are based on well-established and routinely used bELISA protocols (Blitvich et al., 2003a,b; Hall et al., 1995).

\subsection{Traditional influenza A virus serological assays}

The AGID assay was performed according to a standard protocol (Woolcock, 2008). The antigen used in the assay was derived from the conserved influenza A virus nucleoprotein and matrix protein. Reagents were provided by the National Veterinary Service Laboratories (NVSL) in Ames, IA. The hemagglutination-inhibition (HI) and neuraminidase-inhibition (NI) tests were performed at the NVSL following standard protocols (Killian, 2008; Pedersen, 2008). 
Table 1

Percent concordance between the AGID assay and the bELISA for antibody detection in serum collected from influenza A virus experimental infection studies.

\begin{tabular}{|c|c|c|c|c|}
\hline Species-subtype & No. of serum samples & $\%$ positive AGID ( $95 \% \mathrm{CI}$ ) & \% positive bELISA ( $95 \% \mathrm{CI}$ ) & $\%$ concordance $(95 \% \mathrm{CI})$ \\
\hline Raccoon-H3N2 & 41 & $29.3(15.3,43.2)$ & $41.5(26.4,56.5)$ & $87.8(77.8,97.8)$ \\
\hline Raccoon-H4N8 & 78 & $24.4(14.8,33.9)$ & $26.9(17.1,36.8)$ & $97.4(93.9,100.9)$ \\
\hline Mallards-H4N6 & 48 & $33.3(20.0,46.7)$ & $45.8(31.7,59.9)$ & $81.3(70.2,92.3)$ \\
\hline Mallards-H8N4 & 84 & $48.8(38.1,59.5)$ & $83.3(75.4,91.3)$ & $65.5(55.3,75.6)$ \\
\hline Total & 251 & & & $82.1(77.3,87.6)$ \\
\hline
\end{tabular}

\subsection{Comparison of detection levels between bELISA and the AGID assay}

To assess further the sensitivity of the bELISA, 18 arbitrarily selected serum samples were used in a dilution experiment. Briefly, sera from animals challenged experimentally (mallard and raccoon) and three naturally exposed raccoons were tested at multiple dilutions by bELISA and AGID assay. Serum samples were diluted serially two-fold, with a starting dilution of $1: 10$ and a final dilution of 1:1280. Each serum sample was also tested undiluted. The last dilution in which the binding of the monoclonal antibody was inhibited significantly was considered the detection threshold for the bELISA and the last dilution in which a precipitation line was observed was considered the detection threshold for the AGID assay.

\subsection{Statistical analysis}

The efficacy of the bELISA was compared to the AGID assay using sera from experimentally and mock challenged raccoons and mallards. Each sample analyzed by both the bELISA and AGID assay was scored as to whether they agreed or disagreed that antibodies had been detected. Using these data, percent concordance with 95\% confidence intervals were calculated to estimate the agreement between the two assays. A kappa statistic $(\kappa)$ was used to measure the strength of this agreement as a chancecorrected proportional agreement ranging from 0 (indicating no agreement above that expected by chance) to 1 (indicating perfect agreement) (Landis and Koch, 1977). Next a logistic regression (Hosmer and Lemeshow, 2000) was used to compare detection rates of antibodies to influenza A virus in sera from experimentally challenged raccoons and mallards (both negative control and infected animals) using the bELISA and AGID assay. The response variable in this model was binary, with one indicating antibodies to influenza A virus were detected and zero indicating they were not detected. Explanatory variables used in the analysis included method (bELISA or AGID assay), species (mallard or raccoon) and days post-inoculation. PROC LOGISTIC in SAS $^{\circledR}$ was used to analyze the data in models representing different combinations of the explanatory variables with and without interactions (Allison, 1999). The logistic regression model best explaining the data was selected using information-theoretic model selection based on corrected Akaike Information Criterion (AICc) and Akaike weights (Burnham and Anderson, 2003). In the selected model, odds ratios for the method was computed to determine whether one method performed better than the other and maximum-rescaled $R^{2}$ was used to estimate how much variation in the data was explained by the logistic regression model (Hosmer and Lemeshow, 2000).

\section{Results}

\subsection{Determining the diagnostic criterion}

To determine the positive threshold for the bELISA 245 serum samples were used from Maryland from six wild bird (Canada goose, great blue heron, dark-eyed Junco, American white pelican, rock dove, mallard, and wood duck) and five wild mammal species (American black bear, groundhog, raccoon, red fox and white-tailed deer) negative for antibodies to influenza A virus by the AGID assay. However, twenty-four of these serum samples (Canada goose, mallard and wood duck) inhibited the binding of the monoclonal antibody by $\geq 30 \%$ using the bELISA. Eight of these samples were submitted to the NVSL for $\mathrm{HI}$ and NI testing, the remaining 16 samples had insufficient volumes. Of these, seven (87.5\%) yielded evidence of antibodies to influenza A virus by the HI and/or NI test. These samples and the remaining serum samples that had inhibited the binding of the antibodies to influenza A virus monoclonal antibody in the bELISA were therefore removed from the analysis for determining the positive threshold. The mean inhibition value of the 222 remaining negative serum samples was $4.3 \%$ with a standard deviation of $13.5 \%$. Therefore, an inhibition value of $32 \%$ (mean inhibition value \pm 2 S.D.) was selected as the diagnostic criterion to indicate the presence of antibodies to influenza A virus by the bELISA.

\subsection{Evaluation of the bELISA using serum samples from experimentally challenged animals}

The diagnostic efficacy of the bELISA was evaluated using serum samples obtained from experimentally challenged raccoons and mallards (Table 1). In the experiments performed with raccoons, all four raccoons challenged with a H3N2 subtype seroconverted and three of the eight raccoons challenged with a H4N8 subtype seroconverted, which was demonstrated in the bELISA as well as the AGID assay. In all instances, the bELISA detected the antibodies to influenza A virus either prior to or concurrently with the AGID assay post-inoculation. Antibodies to influenza A virus were not detected in any of the negative control raccoons by either bELISA or the AGID assay. The concordance between the AGID assay and bELISA in these experimental raccoon infection studies was $94.1 \%$ (95\% CI = 89.9, 98.3; Table 1) with $\kappa=0.858$ (95\% CI = 0.757, 0.959), indicating strong agreement between AGID assay and bELISA for this species.

Seven of 48 (14.6\%) serum samples collected from mallards inoculated with influenza A virus subtype H4N6 tested positive by bELISA and negative by the AGID assay. One serum sample was positive by the AGID assay but negative by bELISA. For the second cohort of mallards inoculated with influenza A virus subtype H8N4, 29 of the $84(34.5 \%)$ serum samples tested positive for the antibodies to influenza A virus by the bELISA and negative by the AGID assay. The concordance between the AGID assay and bELISA in these experimental mallard infection studies was $71.2 \%(95 \% \mathrm{CI}=63.5$, 78.9; Table 1 ), with $\kappa=0.468$ ( $95 \% \mathrm{CI}=0.342,0.594)$, indicating only moderate agreement between AGID assay and bELISA for this species. This agreement for mallards was significantly lower than for the inoculated raccoons.

For the 251 mallard and raccoon serum samples evaluated from these experimental inoculations, the overall concordance between the AGID assay and bELISA was $82.1 \%(95 \% \mathrm{CI}=77.3,86.8$; Table 1$)$ with $\kappa=0.653(95 \% \mathrm{CI}=0.565,741)$, which indicated moderate agreement between AGID assay and bELISA.

In the logistic regression analysis, a model incorporating the variables: species (mallards versus raccoons), method (AGID assay versus bELISA), days post-inoculation and the interaction between 
species and method was selected based on minimum AICc over other models having fewer effects. The selected model had an Akaike weight of 0.91 , indicating it explained the data substantially better than other models in the set of models examined. This model also explained $36.3 \%$ of the variation in the data. Based on odds ratios, this model indicated that overall the bELISA was $2.5(95 \%$ $\mathrm{CI}=1.6,3.8)$ times more likely than the AGID assay to detect antibodies to influenza A virus in experimentally challenged animals. However, the odds ratio for mallards indicated that the bELISA was $5.8(95 \% \mathrm{CI}=2.9,11.7)$ times more likely than the AGID assay to detect antibodies, whereas the odds ratio for raccoons indicated that the bELISA was only $1.3(95 \% \mathrm{CI}=0.76,2.4)$ times more likely than the AGID assay to detect antibodies. In addition, the odds ratio for raccoons was not statistically different than 1 (i.e., no difference), based on the $95 \%$ confidence intervals. Thus, when compared to the AGID assay, the bELISA was more efficacious when used to assay serum samples from mallards as compared to raccoons and this effect of detectability between the two methods appeared to be greater in mallards at earlier time points in the infection period whereas the effect in raccoons was more constant across the infection period (Fig. 1).
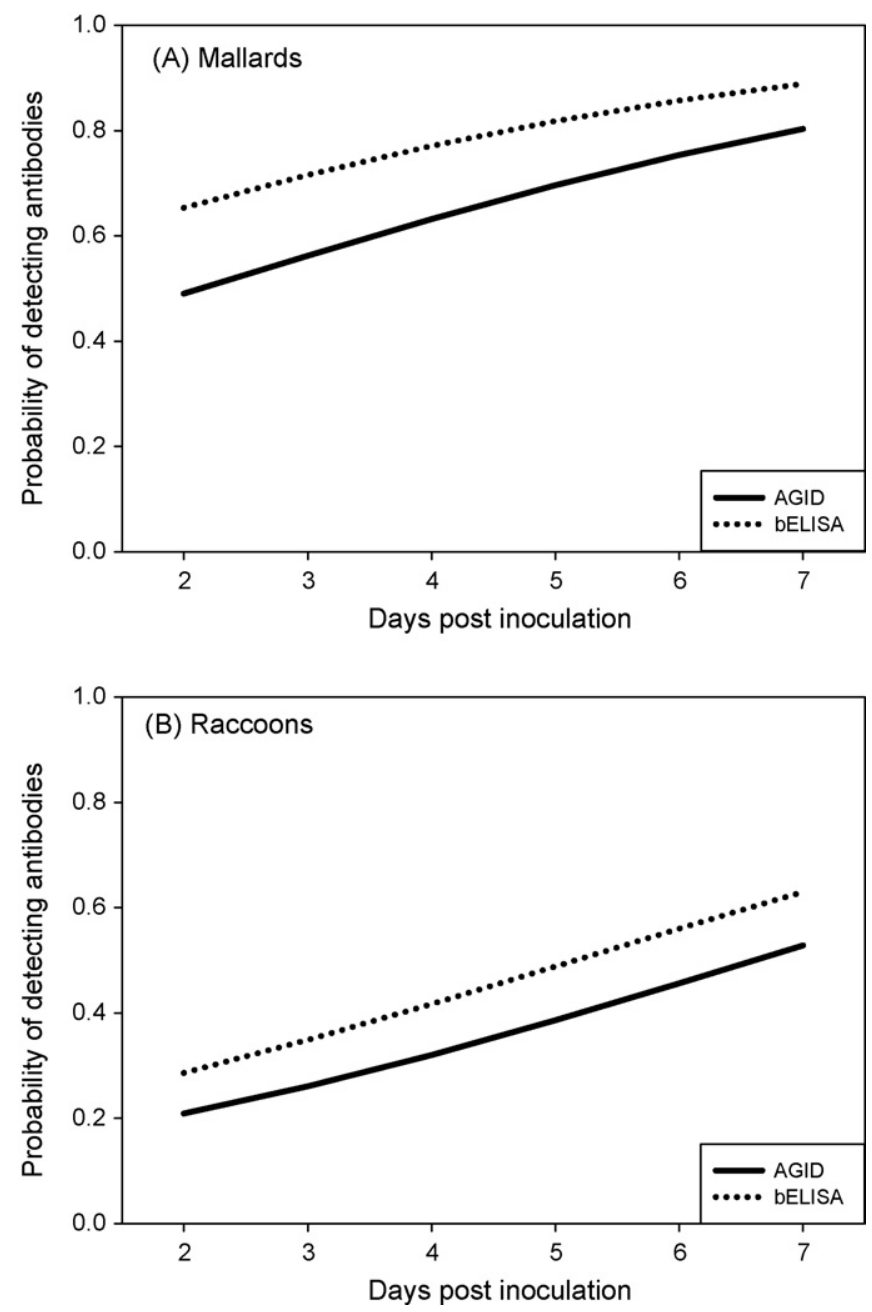

Fig. 1. Probability of detecting antibodies to influenza A virus using the bELISA and AGID assay on days post-inoculation for experimentally challenged (A) mallards and (B) raccoons based on a logistic regression model that included effects of species (mallard versus raccoon), method (bELISA versus AGID assay) and days postinoculation. The dotted lines represent probability of detecting antibodies based on bELISA and solid lines represent probability of detecting antibodies based on AGID assay.
Table 2

Determination of the antibodies to influenza A virus detection levels in selected mallard and raccoon serum samples by bELISA and the AGID assay.

\begin{tabular}{lcl}
\hline Sample ID & Blocking ELISA dilution & AGID dilution \\
\hline${ }^{a}$ M1 & $1: 640$ & $1: 10$ \\
M2 & $1: 640$ & $1: 1$ \\
M3 & $1: 640$ & $1: 1$ \\
M4 & $\geq 1: 1280$ & $1: 1$ \\
M5 & $\geq 1: 1280$ & $1: 1$ \\
M6 & $1: 640$ & $1: 1$ \\
M7 & $1: 640$ & $1: 1$ \\
M8 & $1: 160$ & $1: 1$ \\
M9 & $\geq 1: 1280$ & $1: 1$ \\
${ }^{b}$ R & $\geq 1: 1280$ & $1: 1$ \\
R1 & $1: 640$ & $1: 1$ \\
R2 & $\geq 1: 1280$ & $1: 10$ \\
R3 & $\geq 1: 1280$ & $1: 160$ \\
R4 & $1: 320$ & $1: 10$ \\
${ }^{c} N R 1$ & $\geq 1: 1280$ & $1: 10$ \\
NR2 & $1: 40$ & $1: 1$ \\
NR3 & $\geq 1: 1280$ & $1: 1$ \\
\hline
\end{tabular}

${ }^{a}$ M: influenza A virus experimentally challenged mallard.

b R: influenza A virus experimentally challenged raccoon.

c NR: influenza A virus naturally exposed raccoon.

\subsection{Utility of the bELISA in determining natural exposure to influenza $A$ virus in wild populations}

Serum samples $(n=492)$ were tested for antibodies to influenza A virus using the AGID assay and bELISA. The concordance between the two tests was $93.5,87.7,87.5$ and $63.5 \%$ in rock doves $(n=31)$, feral swine $(n=122)$, raccoons $(n=16)$ and Canada geese $(n=323)$, respectively. To investigate discrepancies between the bELISA and AGID assay results, a subset of serum samples from each species was sent to the NVSL in Ames, IA for standard HI and NI confirmation/subtyping testing. For samples that were positive by bELISA but negative by the AGID assay, the HI and/or NI tests agreed with the bELISA 26/28 (92.3\%) times. In samples that tested negative by bELISA but positive by AGID assay, the HI and/or NI tests agreed with the bELISA 2/9 (22.2\%) times. Canada geese were exposed to HA subtypes (H1, H4, H5 and H6) and NA subtypes (N1, N2, N4, N6 and N8). Rock doves were exposed to HA subtypes (H1 and $\mathrm{H} 4$ ) and NA subtypes (N7 and N8). Feral swine were exposed to the NA subtype N2. No HA subtypes were identified from these swine samples. Raccoons were exposed to HA subtype H3.

\subsubsection{Comparison of detection levels between bELISA and the AGID assay}

The last dilution in which the binding of the monoclonal antibody was significantly inhibited for the bELISA was $\geq 1: 1280$, and the last dilution that presented a precipitation line for the AGID assay was 1:160 (Table 2).

\section{Discussion}

There are several diagnostic assays available for influenza A virus serological studies (OIE, 2008). However, each diagnostic assay has limitations. The HI is used to confirm and subtype samples that have tested positive for influenza A virus antibodies using an assay used for screening. The HI test is expensive, subtype-specific, requires a large volume of serum which is problematic when working with small passerines or mammals such as rodents. The HI on occasion requires modification when testing mammals and some wild bird species for exposure to avian derived influenza A virus (Lu et al., 1982; Kida et al., 1994; Ninomiya et al., 2002; Cattoli and Capua, 2007; VanDalen et al., 2009). Therefore, it is cost prohibitive and is not typically used for large-scale serological surveys for screening samples for influenza A virus in mammals and some wild bird 
species. The AGID assay has been the traditional screening assay or 'gold standard' when dealing with a large number of serum samples for antibodies to influenza A virus during surveillance studies in poultry. While this test is able to detect antibodies to all influenza $A$ virus subtypes, it is very subjective and prone to misinterpretation, requires ten times the volume of sera compared to the bELISA, and may not be ideal for use with all species (Higgins, 1989). The previously described bELISAs for the detection of antibodies to influenza A virus require the purification of the monoclonal antibodies to influenza A virus from hybridoma cells and expression and purification of the recombinant nucleoprotein antigen from insect cells infected with recombinant baculoviruses (de Boer et al., 1990; Zhou et al., 1998; Shafer et al., 1998; Starick et al., 2006). These additional steps are cost and time prohibitive and may not be feasible in many laboratories. The efficacy of most ELISAs used to detect antibodies to influenza A virus in wildlife have not yet been thoroughly evaluated. Recently, a commercial ELISA kit (Multi-S kit, IDEXX, Westbrook, $\mathrm{ME}$ ) has been made available for screening mallard, goose, turkey, chicken and ostrich serum for antibodies to influenza A virus, but the kit is costly and has not been thoroughly evaluated for testing mammal serum.

Due to the constraints mentioned above, our laboratory sought to develop a bELISA using commercially available reagents that would be rapid, cost effective, and could be used for multiple species. Conventionally, when attempting to validate a new assay, it is a common practice to statistically compare results from the new assay to a 'gold standard' assay (defined as assessing infection status with certainty) to estimate the accuracy (i.e., sensitivity and specificity) of the new assay. Since the AGID assay used to screen influenza A virus antibodies in poultry has not yet been thoroughly evaluated for wildlife and has known limitations, the bELISA was compared to the AGID assay, but without a 'gold standard'. Therefore, sensitivity and specificity were not calculated. Instead percent concordance was calculated, which was $71.2 \%(95 \% \mathrm{CI}=63.5,78.9$; Table 1) for avian species and $94.1 \%(95 \% \mathrm{CI}=89.9,98.3$; Table 1) for the mammalian species evaluated. Based on logistic regression the variation found in the data from experimental infections was best explained in a model incorporating species, method, days postinoculation, and interaction between species and method. In an attempt to evaluate the discrepancies between the bELISA and AGID assay, a subset of samples $(n=37)$ was sent to the NVSL, Ames, IA for $\mathrm{HI}$ testing. While it would have been advantageous to confirm and subtype all of the samples using the HI assay, this was not possible because of budget constraints (estimated cost \$37,000). From our subset of samples, the HI test and/or NI test agreed with the bELISA in $67 \%(24 / 36)$ of the samples and agreed with the AGID assay in only $33 \%(12 / 36)$ of the samples. These data provide additional evidence that the bELISA is more reliable than the AGID assay for the detection of influenza A antibodies. In addition, some studies have shown the standard HI protocol (Killian, 2008) can produce falsenegative results for certain mammal and avian samples (Lu et al., 1982; Kida et al., 1994; Ninomiya et al., 2002; Cattoli and Capua, 2007; VanDalen et al., 2009). Therefore, estimates of agreement above may be underestimated.

There are several distinct advantages in using this bELISA for large-scale, multi-species serological surveys. This bELISA is not subtype-specific, it is more objective than some traditional techniques (i.e., AGID assay), it requires small volumes of sera and can be performed using commercially available reagents. Because the reagents can be used at very high optimal dilutions $(143 \mathrm{mg} / \mathrm{ml}$ for the nucleoprotein antigen and $50 \mathrm{mg} / \mathrm{ml}$ for the monoclonal antibody) and test samples at low volumes, the bELISA is an inexpensive and specimen conservative screening assay. In addition, when the detection levels were compared between the bELISA and the AGID assay it was shown that the likelihood of detecting serum samples with low titers of antibodies to influenza A virus is improved when using the bELISA as compared to the AGID assay (Table 2). It was also shown that the bELISA will detect antibodies to influenza A virus earlier to post-infection in experimentally challenged raccoons and mallards, when compared to the AGID assay (Fig. 1). The bELISA was able to detect antibodies to influenza $A$ virus in a number of different avian and mammalian species exposed to multiple influenza $A$ virus subtypes.

\section{Conclusion}

In summary, a sensitive, inexpensive, objective, speciesindependent bELISA platform has been developed, that can be performed in most laboratories and can screen for a variety of influenza A virus-specific subtypes. For these reasons this assay is ideal for large-scale serological studies in multiple domestic and wild mammalian and avian species.

\section{Acknowledgments}

We are indebted to T. Anderson (National Wildlife Research Center $\{$ NWRC $\}$ ) for logistical assistance and the Texas Wildlife Services (WS), Pennsylvania WS for assistance with sample collection. We also thank the many public and private land stewards that allowed us to access to their lands for trapping. In addition, we thank R.A. Bowen (Colorado State University), C. Driscoll (Maryland Department of Natural Resources) and J. Hall (National Wildlife Health Center $\{\mathrm{NWHC}\}$ ) for providing sera for evaluation. Funding for this work was provided by the United States Department of Agriculture. The use or mention of products in this article does not imply endorsement on the part of the United States Government.

\section{References}

Allison, P.D., 1999. Logistic Regression using the SAS ${ }^{\circledR}$ System: Theory and Application. SAS Institute Inc., Cary, NC.

Beard, C.W., 1970. Avian influenza antibody detection by immunodiffusion. Avian Dis. 14, 337-341.

Blitvich, B.J., Bowen, R.A., Marlenee, N.L., Hall, R.A., Bunning, M.L., Beaty, B.J., $2003 a$. Epitope blocking enzyme-linked immunosorbent assays for detection of West Nile virus antibodies in domestic mammals. J. Clin. Microbiol. 41, 2676-2679.

Blitvich, B.J., Marlenee, N.L., Hall, R.A., Calisher, C.H., Bowen, R.A., Roehrig, J.T., Komar, N., Langevin, S.A., Beaty, B.J., 2003b. Epitope-blocking enzyme-linked immunosorbent assays for the detection of serum antibodies to West Nile virus in multiple avian species. J. Clin. Microbiol. 41, 1041-1047.

Burnham, K.P., Anderson, D.R., 2003. Model Selection and Multi-model Inference. Springer, New York, NY.

Cattoli, G., Capua, I., 2007. Diagnosing avian influenza in the framework of wildlife surveillance efforts and environmental samples. J. Wildl. Dis. Suppl. 43, S35-S39.

de Boer, G.F., Back, W., Osterhaus, A.D., 1990. An ELISA for detection of antibodies against influenza A nucleoprotein in humans and various animal species. Arch. Virol. 115, 47.

Fenner, F.J., Gibbs, E.P., Murphy, F.A., Rott, R., Studdert, M.J., White, D.O., 1993. Orthomyxoviridae. In: Veterinary Virology, 2nd ed. Academic Press, Inc., San Diego, CA, pp. 511-522.

Fouchier, R.A., Munster, V., Wallensten, A., Bestebroer, T.M., Herfst, S., Smith, D. Rimmelzwaan, G.F., Olsen, B., Osterhaus, A.D., 2005. Characterization of a novel influenza A virus hemagglutinin subtype (H16) obtained from black-headed gulls. J. Virol. 79, 2814-2822.

Hall, J.S., Minnis, R.B., Campbell, T.A., Barras, S., DeYoung, R.W., Pablionia, K., Avery, M.L., Sullivan, H., Clark, L., McLean, R.G., 2008a. Influenza exposure in United States feral swine populations. J. Wildl. Dis. 44, 362-368.

Hall, J.S., Bentler, K.T., Landolt, G., Elmore, S.A., Minnis, R.B., Campbell, T.A., Barras, S.C., Root, J.J., Pilon, J., Pabilonia, K., Driscoll, C., Slate, D., Sullivan, H., McLean, R.G., 2008b. Influenza infection in wild raccoons. Emerg. Infect. Dis. 14, 1842-1848.

Hall, R.A., Broom, A.K., Hartnett, A.C., Howard, M.J., Mackenzie, J.S., 1995. Immunodominant epitopes on the NS1 protein of MVE and KUN viruses serve as targets for a blocking ELISA to detect virus-specific antibodies in sentinel animal serum. J. Virol. Methods 51, 201-210.

Higgins, D.A., 1989. Precipitating antibodies of the duck (Anas platyrhynchos). Comp. Biochem. 93, 135-144.

Hosmer, D.W., Lemeshow, S., 2000. Applied Logistic Regression, 2nd ed. John Wiley and Sons, New York, NY.

Kida, H., Ito, T., Yasuda, J., Shimizu, Y., Itakura, C., Shortridge, K.F., Kawaoka, Y., Webster, R.G., 1994. Potential for transmission of avian influenza viruses to pigs. J. Gen. Virol. 75, 2183-2188. 
Killian, M.L., 2008. Hemagglutination assay for the avian influenza virus. In: Spackman, E. (Ed.), Avian Influenza Virus. Humana Press, Totowa, NJ, pp. 47-52.

Landis, J.R., Koch, G.C., 1977. The measurement of observer agreement for categorical data. Biometrics 33, 159-174

Lu, B.L., Webster, R.G., Hinshaw, V.S., 1982. Failure to detect hemagglutinationinhibiting antibodies with intact avian influenza virions. Infect. Immun. 38, 530-535.

Ninomiya, A., Takada, A., Okazaki, K., Shortridge, K.F., Kida, H., 2002. Seroepidemiological evidence of avian H4, H5, and H9 influenza A virus transmission to pigs in southeastern China. Vet. Microbiol. 88, 107-114.

World Organization for Animal Health (OIE), 2004 December 2008, posting date. Avian Influenza. In: Manual of Diagnostic Tests and Vaccines for Terrestrial Animals, 14th ed., OIE, Paris, France (Chapter 2.7.12), http://www.oie.int/Eng/Normes/Mmanual/A_00037.htm.

Palese, P., Shaw, M.L., 2007. Orthomyxoviridae: the virus and their replication. In: Fields, B.N., Knipe, D.M., Howley, P.M. (Eds.), Fields Virology. Lippincott-Raven, Philadelphia, PA, pp. 1647-1689.

Paltrinieri, S., Spagnolo, V., Giordano, A., Martin, A.M., Luppi, A., 2008. Influenza virus type A serosurveys in cats [letter]. Emerg. Infect. Dis., http://www.cdc.gov/ EID/content/13/4/662.htm.

Pedersen, J., 2008. Neuraminidase-inhibition assay for the identification of influenza A virus neuraminidase subtype or neuraminidase antibody specificity. In: Spackman, E. (Ed.), Avian Influenza Virus. Humana Press, Totowa, NJ, pp. 67-76.

Root, J.J., McLean, R.G., Slate, D., MacCarthy, K.A., Osorio, J.E., 2008. Potential effect of prior raccoonpox virus infection in raccoons on vaccinia-based rabies immunization. BMC Immunol. 9, 57

Shafer, A.L., Katz, J.B., Eernisse, K.A., 1998. Development and validation of a competitive enzyme-linked immunosorbent assay for detection of type A influenza antibodies in avian sera. Avian Dis. 42, 28-34.
Spackman, E., 2008. A brief introduction the avian influenza virus. In: Spackman, E. (Ed.), Avian Influenza Virus. Humana Press, Totowa, NJ, pp. 1-6.

Starick, E., Werner, O., Schirrmeier, H., Köllner, B., Riebe, R., Mundt, E., 2006. Establishment of a competitive ELISA (cELISA) system for the detection of influenza $A$ virus nucleoprotein antibodies and its application to field sera from different species. J. Vet. Med. B: Infect. Dis. Vet. Public Health 53, 370-375.

Toth, T.E., Norcross, N.L., 1981. Precipitating and agglutinating activity in duck antisoluble protein immune sera. Avian Dis. 25, 338-352.

U.S. Departments of Interior, Agriculture, and Health and Human Services. An Early Detection System for Highly Pathogenic H5N1 Avian Influenza in Wild Migratory Birds U.S. Interagency Strategic Plan, 2006.

VanDalen, K., Shriner, S., Sullivan, H., Root, J.J., Franklin, A., 2009. Monitoring wild mammal exposure to avian influenza viruses. Mammal Rev., in press, doi:10.1111/j.1365-2907.2009.00144.x.

Webster, R.G., Bean, W.J., Gorman, O.T., Chambers, T.M., Kawaoka, Y., 1992. Evolution and ecology of influenza A viruses. Microbiol. Rev. 56, 152-179.

Woolcock, P.R., 2008. Avian influenza virus isolation and propagation in chicken eggs. In: Spackman, E. (Ed.), Avian Influenza Virus. Humana Press, Totawa, NJ, pp. 35-46.

World Health Organization (WHO), December 2008, posting date. 14th ed., OIE, Paris, France, http://www.who.int/csr/disease/avian_influenza/country/cases table_2008_12_16/en/index.html.

Wu, R., Hu, S., Xiao, Y., Li, Z., Shi, D., Bi, D., 2007. Development of indirect enzymelinked immunosorbent assay with nucleoprotein as antigen for detection and quantification of antibodies against avian influenza virus. Vet. Res. Commun. 31 631-641.

Zhou, E.M., Chan, M., Heckert, R.A., Riva, J., Cantin, M.F., 1998. Evaluation of a competitive ELISA for detection of antibodies against avian influenza virus nucleoprotein. Avian Dis. 42, 517-522. 